\title{
25 Research Square \\ The mechanism of cartilage regeneration by buffy coat and the pre-clinical study
}

\section{Longhao Jin ( $\nabla$ jlh0423@sina.com )}

Yanbian Hospital: Yanbian University Hospital https://orcid.org/0000-0003-4435-2967

\section{Hang Su}

Yanbian Hospital: Yanbian University Hospital

\section{guo Song}

Yanbian Hospital: Yanbian University Hospital

\section{Kumar Gurung}

Community Hospital

\section{Shouyong FU}

Yanbian Hospital: Yanbian University Hospital

\section{GuangWen Jin}

Yanbian Hospital: Yanbian University Hospital

Hao Mei

Yanbian Hospital: Yanbian University Hospital

\section{Xu Wang}

Yanbian Hospital: Yanbian University Hospital

\section{Research Article}

Keywords: Cartilage Repair, Buffy Coat, Mesenchymal Stem Cells, Osteoarthritis

Posted Date: February 22nd, 2022

DOI: https://doi.org/10.21203/rs.3.rs-98538/v3

License: (a) (i) This work is licensed under a Creative Commons Attribution 4.0 International License.

Read Full License 


\section{Abstract}

\section{Background}

Autologous bone marrow buffy coat transplantation possesses obvious advantages in the therapy of cartilage defects. However, there is no definite research on the specific effective components of bone marrow buffy coat and its mechanism of cartilage regeneration. Moreover, bone marrow buffy coat is difficult to fix onto the damaged cartilage area. We assessed the effect of using hyaluronic acid(HA) as a gel scaffold mixed with autologous bone marrow buffy coat to fix cartilage defect.

\section{Methods and Materials}

We extracted the bone marrow from the anterior superior iliac crest of rabbit, centrifuged to obtain buffy coat, and analyzed the components of buffy coat by ELISA. Buffy coat+fibrin/HA group, MSC+fibrin/HA group, MSC+TGF- $\beta+$ fibrin/HA group were cultured in vitro and observed by staining. In addition, we made damage to the femoral condyle of rabbits, and divided them into groups: HA group, buffy coat group, buffy coat with HA group. Each group was assessed for cartilage regeneration by visual observation, histological at 4 weeks and 8 weeks, and biochemical analysis at 8 weeks postoperatively. One-way ANOVA and LSD were used for statistic analysis.

\section{Results}

Buffy coat have a variety of growth factors, inflammatory factors and anti-inflammatory factors that stimulate the MSCs' regeneration. Buffy coat can differentiate into cartilage without TGF- $\beta$ stimulation in vitro. The cartilage regeneration ability of buffy coat and buffy coat+HA is strong, and the combination of buffy coat and gel scaffold HA can make cartilage formation ability more stable in vivo.

\section{Conclusion}

MSC and cytokines in buffy coat synergistically promote cartilage regeneration. Gel scaffold HA enhances the effect of buffy coat on cartilage attachment and regeneration of cartilage defects.

\section{Introduction}

Injuries to the articular cartilage are challenging to repair because cartilage possesses a limited capacity for self-repair [1]. Because articular cartilage is avascular, aneural, and alymphatic, the treatment of articular cartilage defects faces many clinical challenges[2]. Low cell mobility due to surrounding matrix and a limited number of progenitor cells could be another contributing factor. Remarkable efforts have been made to repair cartilage defects. Several techniques concerning implantation of different kinds of cells or tissues aiming to instance autologous chondrocyte implantation and mosaicplasty were developed to restore the normal structure and function of cartilage [3]. These techniques have been demonstrated to be valid in enhancing cartilage repair. However, the regeneration of hyaline cartilage has not been adequately accomplished. 
Numerous studies have demonstrated the therapeutic potential of mononuclear cells (MNCs) myocardial infarction, and cartilage injury [3-6]. In addition, BMSCs constitute $0.001-0.01 \%$ of mononuclear cells in bone marrow aspirate after undergoing density gradient centrifugation to remove platelets, granulocytes, red blood cells, and immature myeloid precursors [7]. Chondrogenesis of MSC requires stimulation of a variety of growth factors, such as transforming growth factor- $\beta 1$ (TGF- $\beta 1$ ) and platelet-derived growth factor (PDGF) [8, 9]. Jin et al. (2011) first observed that the buffy coat containing monocytes, lymphocytes, macrophages, platelets, and various cytokines displayed satisfactory cartilage regeneration [10],as the achieved number of MSCs in the buffy coat are higher than that of the bone marrow stimulation (100 times). The buffy coat of $4 \mathrm{ml}$ bone marrow contained $2400 \mathrm{MSCs}$ and $1 * 10^{7} \mathrm{MNC}$. Buffy coat possesses an acceptable cartilage differentiation capability in vitro [11]. However, the mechanism of cartilage regeneration of the buffy coat is unclear and buffy coat is difficult to fix onto the damaged cartilage area.

During preparation, the use of a liquid buffy coat during surgery did not attach cartilage defects as effectively as expected. The analysis of the cytokine components of buffy coat provides a perspective on how buffy coat can repair cartilage. And the combination of buffy coat with hyaluronic acid (HA) and polygly

colic acid (PGA) provides a solution for its clinical application.

HA is a biodegradable, biocompatible, non-toxic, non-immunogenic, and non-inflammatory linear polysaccharide [12]. In cartilage, HA chains are used as aggregated anchors of aggrecan monomers, and play a pivotal role in assuring cartilage hydration, joint surface lubrication, and synovial fluid viscosity $[13,14]$.

Hence, in the present study, the buffy coat components and chondrogenic ability of the buffy coat in vitro and cartilage regeneration of the mixture of HA and buffy coat of in vivo was analyzed. This study may provide an important theoretical and experimental basis for the use of buffy coat in regenerating cartilage.

\section{Materials And Methods}

\subsection{Materials}

Healthy 16-week-old (range of weight, 3.0-3.5 kg) New Zealand white rabbits were purchased from the Experimental Animal Center of Yanbian University (Yanji, China). All of the rabbits were housed during the period of experiment under the conditions of a $12: 12 \mathrm{~h}$ light/dark cycle at $27^{\circ} \mathrm{C}$. A total of $42 \mathrm{rabbits}$ were used in the experiment.

\subsection{Component analysis of buffy coat}

We randomly extracted 8 buffy coat supernatant samples from the in vitro and in vivo buffy coat groups for composition analysis. Moreover, $2 \mathrm{ml}$ of bone marrow was extracted from the anterior superior iliac 
crest of rabbits using a $20 \mathrm{ml}$ syringe containing $2 \mathrm{ml}$ of $0.9 \%$ sodium chloride solution. The collected bone marrow was placed in a tube containing Ficoll (17-1440-02; GE Healthcare, Sweden), and centrifuged at $1000 \mathrm{~g}$ for $30 \mathrm{~min}$ at $4^{\circ} \mathrm{C}$ [10]. We extract $0.5 \mathrm{ml}$ buffy coat into $1.5 \mathrm{ml}$ tube and centrifuge again at $500 \mathrm{~g}$ at $4^{\circ} \mathrm{C}$ for $3 \mathrm{~min} .20 \mu \mathrm{l}$ cells and supernatant mixture were used for in vitro and in vivo experiments.

The analysis of PDGF, TGF- $\beta 1$, vascular endothelial growth factor (VEGF), epithelial growth factor (EGF), insulin like growth factor-1 (IGF-1), fibroblast growth factor-2 (FGF-2), interlukin-1 $\beta$ (IL-1 $\beta$ ), interlukin-6 (IL6), tissue necrosis factor-a (TNF-a), interlukin-4 (IL-4), and interlukin-10 (IL-10) was performed using an enzyme-linked immunosorbent assay (ELISA) kit (Elabscience, Houston, TX, USA), according to the manufacturer's instructions.

\subsection{In vitro study}

The mixing ratio of fibrinogen (Shanghai RAAS Blood Products Co., Ltd., Shanghai, China) to HA (Hunan Jingfeng Pharmaceutical Co., Ltd., Shanghai, China) was 10:1. Cells with density of $5 * 10^{6}$ cells/well were then mixed homogeneously with aprotinin, $60 \mathrm{U} / \mathrm{mL}$ thrombin, fibrin stabilizing Factor XIII, and 50 $\mathrm{mM} \mathrm{CaCl}_{2}$. Then, $250 \mu \mathrm{L}$ of the fibrin/HA mixture was dropped into an empty Petri dish to form a gel [15].a rabbit was used to extract MSC as the MSC group and the MSC+TGF- $\beta$ group, and 5 rabbits were used to extract buffy coat as buffy coat group.

Buffy coat was re-suspended in an a-minimum essential medium (a-MEM) supplemented with $1 \%$ antibiotic-antimycotic and 10\% fetal bovine serum (FBS). Buffy coat was cultured in an incubator with $5 \%$ $\mathrm{CO}_{2}$ at $37^{\circ} \mathrm{C}$. After 6 days, non-adherent cells were removed and adherent cells were further cultured. After 12 days of cultivation, the adherent MSCs were retrieved by trypsin treatment and re-cultivated at density of $3 * 10^{5}$ cells/plate $(100 \mathrm{~mm})$ for expansion. The second-generation cells were harvested into fibrin/HA composite gel ( $\left.5 * 10^{6} \mathrm{MSCs} / \mathrm{scaffold}\right)$. After $90 \mathrm{~min}$ of incubation, they were transferred into a chondrogenic medium consisting of DMEM with $100 \mathrm{U} / \mathrm{mL}$ penicillin, $100 \mu \mathrm{g} / \mathrm{mL}$ streptomycin, 100 $\mu \mathrm{g} / \mathrm{mL}$ pyruvate, $40 \mu \mathrm{g} / \mathrm{mL}$ proline, $50 \mu \mathrm{g} / \mathrm{mL}$ l-ascorbicacid-2-phosphate, $1 \%$ ITS, $100 \mathrm{nM}$ dexamethasone[16]. In the MSC + TGF- $\beta$ group, TGF- $\beta 1$ was added into the chondrogenic medium until the final concentration was $10 \mathrm{ng} / \mathrm{ml}[17]$. The bone marrow aspirates $(2 \mathrm{ml})$ were obtained aseptically from the iliac crest, and buffy coat was obtained by Ficoll gradient centrifugation method.

Buffy coat was seeded into fibrin/HA composite gel ( $\left.5 * 10^{6} \mathrm{MNCs} / \mathrm{scaffold}\right)$. After 90 min of incubation, they were transferred into the chondrogenic medium.

\subsection{In vivo experiment}

Herein, xylazine hydrochloride $(0.1 \mathrm{ml} / \mathrm{kg})$ was injected intramuscularly, and $2 \%$ pentobarbital sodium (2 $\mathrm{ml} / \mathrm{kg}$ ) was injected intraperitoneally into white New Zealand rabbits. After completing anesthetization of these rabbits, the operation was carried out. An arthrotomy was made through a midline longitudinal incision on the lateral parapatellar with the patella dislocated laterally to expose the femoral condyle. A 
surgical drill (5 $\mathrm{mm}$ in diameter) was used for the creation of an osteochondral defect of $2 \mathrm{~mm}$ in depth in the patella groove.

For the in vivo study, we used a PGA (Albany International Inc. Mansfield, MA, USA) scaffold after flattening to cover the cartilage defect. Absorbable sutures were cross-secured simply to prevent the graft from falling into the joint cavity.

The rabbits were randomly divided into three groups: 1. Injecting $20 \mu \mathrm{HA}$ (Hunan Jingfeng Pharmaceutical Co., Ltd., Shanghai, China) after appearance of defect, and the defect was left untreated as a negative control (HA group); 2. Supplemented $20 \mu$ lautologous buffy coat was isolated from the iliac crest (buffy coat group); $3.20 \mu \mathrm{l}$ buffy coat and $20 \mu \mathrm{HA}$ composite were injected into the cartilage defect site (buffy coat+HA group). Regeneration of cartilage was evaluated by macroscopic, histological at 4th and 8th weeks post-operation, and additionally by biochemical analysis at 8th week postoperation. 6 rabbits were used in each group. The design of experiment was conducted based on "Animal research: reporting in vivo experiments: the ARRIVE guidelines" [18].

\subsection{Macroscopic and histological evaluations}

At 4th and 8th weeks post-operation, the rabbits were euthanized by injection of an over-dose of pentobarbital. Femoral condyles were subsequently retrieved. Macroscopic images of the condyles were initially observed; the samples were then embedded into paraffin, sectioned, and processed for performing Safranin-0 staining, Sirius Red staining.

\subsection{Histological scoring}

To minimize the effects of subjective bias, the Safranin-0 stained slides were graded using the Bern Score, consisting of seven categories (range of score: 0-9) [19]. The quality of the regenerated articular cartilage of the defects and a modified version of the histological grading scale system were used for the International Cartilage Repair Society (ICRS) score [20]. The ICRS consists of seven categories, and a score ranging from 0 to 18 was accordingly assigned.

\subsection{Chemical assay of cartilage tissue engineering}

For chemical assays of the repaired tissue, the cartilage specimens were minced by a surgical knife. The harvested tissue was dried and dissolved in papain solution (5 mM L-cysteine, $100 \mathrm{mM} \mathrm{Na}{ }_{2} \mathrm{HPO}_{4}, \mathrm{pH} 6.4$, $5 \mathrm{mM}$ EDTA, and $125 \mathrm{mg} / \mathrm{ml}$ papain type III) at $60^{\circ} \mathrm{C}$ for $24 \mathrm{~h}$, and then centrifuged at $12,000 \mathrm{~g}$ for 10 min. The GAG contents were measured by subjecting the supernatant to 1,9-Dimethylmethylene Blue (DMB) assay.

\subsection{Statistical analysis}

Statistical analysis for comparing the experimental data was carried out by using(one-way ANOVA), and specific inter-data differences between mean values were determined by the LSD test. P-values less than 0.05 were considered statistically significant. 


\section{Results}

\subsection{Composition analysis of buffy coat}

The results showed that buffy coat contains a great number of growth factors that promote cartilage regeneration, and also contains inflammation and anti-inflammation factors. Buffy coat contains growth factors, such as TGF- $\beta 1(1.349 \pm 0.382 \mathrm{ng} / \mathrm{ml})$, PDGF $(1.514 \pm 0.292 \mathrm{ng} / \mathrm{ml}), F G F-2(1.326 \pm 0.818 \mathrm{ng} / \mathrm{ml})$, IGF-1 $(0.195 \pm 0.056 \mathrm{ng} / \mathrm{ml})$, EGF $(0.162 \pm 0.110 \mathrm{ng} / \mathrm{ml})$, VEGF $(0.031 \pm 0.009 \mathrm{ng} / \mathrm{ml})($ Table 1$)$, in addition to other inflammatory factors, including IL-1 $\beta(0.753 \pm 0.138 \mathrm{ng} / \mathrm{ml})$, IL-6 $(0.219 \pm 0.066 \mathrm{ng} / \mathrm{ml})$, TNF-a $(1.031 \pm 0.151 \mathrm{ng} / \mathrm{ml})$, and other anti-inflammatory factors, involving IL-4 $(1.115 \pm 0.657 \mathrm{ng} / \mathrm{ml})$ and IL-10 $(1.969 \pm 0.095 \mathrm{ng} / \mathrm{ml})($ Table 2$)$. It can be seen that numerous factors promoted cartilage regeneration in buffy coat.

Table 1

Growth factors' density in buffy coat

\begin{tabular}{|llllll|}
\hline \multicolumn{5}{|l|}{ Growth factors $(\mathbf{p g} / \mathrm{ml})$} \\
\hline TGF- $\beta 1$ & PDGF & FGF-2 & IGF-1 & EGF & VEGF \\
\hline $1349.4 \pm 381.8$ & $1513.7 \pm 292.9$ & $1326.3 \pm 818.3$ & $195.0 \pm 56.2$ & $162.0 \pm 110.1$ & $32.5 \pm 9.9$ \\
\hline
\end{tabular}

Table 2

Inflammation factors and anti-inflammation factors in buffy coat

\begin{tabular}{|lllll|}
\hline \multicolumn{2}{|l}{ Inflammation factor $(\mathbf{p g} / \mathrm{ml})$} & \multicolumn{3}{c|}{ Anti-inflammation factor $(\mathrm{pg} / \mathrm{ml})$} \\
\hline $\mathrm{IL}-1 \beta$ & $\mathrm{IL}-6$ & TNF-a & IL-4 & IL-10 \\
\hline $753.3 \pm 138.2$ & $219.2 \pm 66.0$ & $1031.2 \pm 151.6$ & $1114.8 \pm 657.4$ & $196.9 \pm 94.8$ \\
\hline
\end{tabular}

\subsection{In vitro evaluation}

After 21 days of culture, it was revealed that the MSC group was generally smaller with blur edges. There was no remarkable change in the size of the buffy coat group and the MSC+TGF- $\beta$ group, both with round and clear edges and high cartilage transparency (Figure 2A). Cells on the fibrin/HA composite gel were stained with Safranin-O, and a significant intergroup difference was noted. On microscopic observation, cells in the outer region of the MSC group displayed differentiation into chondrocytes and production of GAG; however, the central cells did not differentiate into chondrocytes, and apoptosis was noted. On the contrary, in the buffy coat group, cells in the center and surrounding areas displayed active differentiation into chondrocytes and production of GAG. In the MSC+TGF- $\beta$ group, all aspects of cartilage differentiation were active, while the surrounding cartilage was well differentiated, and the central part 
couldn't enter into the culture fluid, resulting in a necrotic cavity in the tissue center (Figure 2B). Analysis of these sections using the Bern score supported this observation. The cells constructed with high cell density showed the increased effect of Safranin-O staining in the buffy coat group and MSC+TGF- $\beta$ group, which demonstrated a rounded cell shape and higher production of extracellular matrix (ECM) (Figure 2B). A significant difference in the Bern score among the buffy coat, MSC+TGF- $\beta$, and MSC groups was noted. The mean score of MSC group was lower than that of the latter two groups, with significant statistical difference. The buffy coat group, however, had a lower mean score than the MSC+TGF- $\beta$ group, but there was no statistical difference. It might be due to the large standard deviation and small sample size. $(n \geq 4 ; \star \star P=0.002 ; * \star \star ~ P<0.001)$ (Figure 3$)$. From this, it can be seen that the MSC contained in buffy coat has a good differentiation ability and various growth factors that can promote the cartilage differentiation.

\subsection{In vivo evaluation}

On the 4th week after operation, regenerated white tissue appeared in the cartilage defect, but the regenerated tissue in the HA group was significantly less than that in the other groups. The surface of the regenerated tissue in the Buffy coat and Buffy coat+HA groups was covered with fibrous tissue. At week 8 , each group had more regenerated tissue than that of week 4 , and the regenerated tissue in the Buffy coat and Buffy coat+HA groups was closer to the surrounding normal cartilage (Figure. 4A).

Safranin-O staining showed that the quality of cartilage regeneration in the HA group was very poor, with defects within the regenerated tissue and very little GAG content, and separate from the subchondral bone at 4 weeks;Buffy coat and Buffy coat+HA groups had abundant GAG regeneration, but the regenerated tissue was immature(Figure. 4B g-I). At 8 weeks, the GAG content in the HA group was still very low, indicating that the regenerated tissue covering the cartilage defect in Figure $4 \mathrm{~d}$ was fibrous tissue rather than cartilage tissue; the Buffy coat group showed that the regenerated tissue was very mature, but the surface was degraded to a certain extent(Figure. 4B $n$ and q).The Buffy coat+HA group produced a large amount of GAG, and the regenerated cartilage also matured(Figure. 4B o and r);The chondrocytes were parallel to the articular surface, and the deep chondrocytes were perpendicular to the articular surface (Figure. 4B o and r).

The ICRS scores were significantly increased in all the groups over time. There was a statistically significant difference in the scores between the HA group and the buffy coat+HA group at 4th week ( $n \geq 5$; $\star * P=0.001)$. The average score in the buffy coat group was notably higher than that in the HA group, which was close to that in the buffy coat+HA group. At 8th week, there was a significant difference in the ICRS scores between the HA group and buffy coat group ( $n \geq 5 ; * * P=0.004 ; * \star \star P<0.001)$. The mean ICRS score was lower in buffy coat compared with buffy coat+HA group, while no significant difference was noted between these two groups (Figure 5). Therefore, the use of HA does not affect the efficacy of buffy coat cartilage regeneration, and HA can bring convenience in operation, (buffy coat fixation in cartilage defects). This experiment provides a theoretical basis for the clinical application of buffy coat. 
In Sirius Red staining, the HA group formed very fine and irregular collagen at week 4,the Buffy coat and Buffy coat+HA groups regenerated relatively more collagen, but the collagen fibers were irregularly arranged(Figure. 6a-f). In the HA group, a small amount of collagen was formed at the 8th week, and the fibers were thicker but irregularly arranged than at the 4th week; at week 8 , the Buffy coat and Buffy coat+HA groups regenerated a large amount of collagen, and the fibers were also thicker and grew perpendicular to the surface, indicating that the regenerated cartilage was very mature (Figure $6 \mathrm{~g}-\mathrm{l})$.

At 8th week post-operation, the content of GAG in the buffy coat group and buffy coat+HA group was significantly higher than that in the HA group, while there was a gap compared with normal cartilage (Figure 7). The standard deviation in the buffy coat group was very large, whereas the standard deviation in the buffy coat+HA group was small, and the regeneration of cartilage was found to be very stable $(n=6$; $\star * P=0.001 ; * \star * P<0.001)$. Therefore, it was found that buffy coat $+H A$ greatly improved the stability of cartilage regeneration, thereby overcoming the problem of the buffy coat to fix to damaged cartilage.

\section{Discussion}

The results of the present study indicated that buffy coat became a suitable material for cartilage regeneration due to its MSC and multiple cytokines. In combination with the gel scaffold HA, buffy coat has the ability to form engineered cartilage independent of additional cytokines and to adhere to cartilage defects. Buffy coat was centrifuged at $500 \mathrm{~g}$ for 3 minutes in the process of use, not only to analyze the composition of buffy coat, but also to collect MSCs, MNCs and other cells.Such a short period of centrifugation with weak centrifugal force was not sufficient to delaminate the cytokines in the buffy coat.

In Safranin-O staining, large amounts of GAG occurred in the cartilage defect staining area, but the subchondral bone did not regenerate (Figure $4 \mathrm{~h} / \mathrm{k}$ and $\mathrm{i} / \mathrm{l}$ ). On the contrary, the thickness of the regenerated cartilage was close to normal cartilage, and the subchondral bone was also regenerated (Figure $4 \mathrm{n} / \mathrm{q}$ and $\mathrm{o} / \mathrm{r}$ ). Thus, the results at 8 weeks were more mature than those at 4 weeks. The ICRS score also support the conclusion. In Sirus Red staining, collagen fibers were numerous but were fine and irregular (Figure 6b/e and c/f), indicating that the regenerated cartilage was immature. On the contrary, the Collagen fiber is thick and with regular direction (Fugure $6 \mathrm{~h} / \mathrm{k}$ and $\mathrm{i} / \mathrm{l}$ ). It indicates that the regenerated cartilage is mature.

Various growth factors are involved in the differentiation of MSC into cartilage. TGF- $\beta 1$ at the range of $0.1-25 \mathrm{ng} / \mathrm{mL}$ is often essential for proper regulation of MSC proliferation, differentiation, and ECM synthesis, while a higher concentration may inhibit the process[21, 22]. All TGF- $\beta 1$ values levels detected in buffy coat were actually within the range suitable for MSC differentiation[23], FGF-2 is normally undetectable in serum or at very low level. A low dose of FGF-2 $(10-100 \mu \mathrm{g} / \mathrm{mL})$ soaked in a highly porous scaffold improved healing of osteochondral lesions[24]. The FGF-2 concentration detected in buffy coat was consistent with the repair concentration of cartilage. In addition, it has been widely reported that IGF1 [25], PDGF[26] and other growth factors play important roles in the process of cartilage repair of buffy 
coat, and the concentration of buffy coat is also within the range of its suitable concentration for cartilage repair.

Generally, VEGF is not found in adult human articular cartilage under physiological conditions and has been reported as one of the critical factors that can cause arthritis. VEGF in the minimum amount can trigger the formation of a new blood vessel and increase blood flow to the site of injury, thereby protecting the proliferated MSCs [27].EGF also negatively regulate cartilage defect repairment. EGF stimulates epithelization and decreases the healing process, in which a high level of EGF causes thinning, loss of integrity, and degradation of the articular cartilage [28]. Since the concentration of EGF is insignificant in the isolated buffy coat, it is reasonable to hypothesize that the chances of losing the integrity and degradation of the articular cartilage are very low, while its' presence can stimulate angiogenesis of endothelial cells and mitosis of mesenchymal stem cells.

In addition, a variety of inflammatory and anti-inflammatory factors are involved in cartilage repairment. IL-1 $\beta$ and TNF- $\alpha$ are present in joints where cartilage is undergoing repairment or regeneration due to injury or disease. In an in vitro study carried out by Schuerwegh et al. [29], it was uncovered that high concentrations of IL-1 $\beta(100 \mathrm{ng} / \mathrm{mL})$ and TNF-a induced apoptosis of bovine chondrocytes. Additionally, no effect of IL-6 was noted on the function of cartilage with respect to chondrocyte apoptosis, viability, and proliferation. To our knowledge, inflammatory cytokines are inevitable after tissue injury or disease. Hence, we hypothesized that the presence of very low concentration $(0.219 \mathrm{ng} / \mathrm{ml})$ of inflammatory cytokines by the buffy coat is negligible [29].

Furthermore, Behrendt et al. found that IL-10 prevented posttraumatic cell death, matrix degradation, and chondrocyte dedifferentiation in mechanically injured cartilage. The IL-10 concentration in our study were close to the that reported by Behrendt et al.[30]. A large number of previous studies have found that IL-4 can inhibit the degradation of proteoglycan in articular cartilage[31]. The concentration of IL-4 obtained in this experiment is $1.115 \mathrm{ng} / \mathrm{mL}$, which may inhibit the degradation of damaged cartilage.

At present, Platelet-rich plasma (PRP) and BMC research has become more prevalent in recent years. Platelet-rich plasma (PRP) is a safe treatment for autologous blood products. It contains high concentrations of platelets that can be treated by minimally invasive arthroscopy. It has also been reported that the repaired cartilage by PRP is fibrocartilage in nature, while the frequency and duration of treatment have not been reported yet[32,33]. It is noteworthy that BMC contains a variety of biologically active molecules and cells, including platelets, lymphocytes, neutrophils, and monocytes, in addition to MSC.A number of studies demonstrated that BMC is a product of PRP with MSC. In fact, BMC and PRP contain different cytokines and growth factors. It was reported that BMC contains several platelets, total monocytes, and CD34 positive cells [34]. Cassano et al. confirmed that BMC is rich in interleukin 1 receptor antagonist (IL-1 ra), which is a natural IL-1 receptor antagonist, and it can treat autoimmune diseases and rheumatoid arthritis[34]. Studies also showed that buffy coat is a BMC without red blood cell (RBC)which could hinders adhesion and proliferation of MSC. Thus, buffy coat has a superior cartilage regeneration capability [11]. Horn et al. also reported that RBCs interferes with adhesion and 
proliferation of MSCs[33]. When buffy coat was extracted, we accidentally scraped numerous RBC samples, in which the quality of tissue-engineered cartilage was not eventually satisfactory.

The same number of MSCs should be inoculated during the experimental comparison, but the number of MSCs in buffy coat is too small and requires a lot of bone marrow, which is impractical during the operation. Therefore, we used $5 * 10^{6} \mathrm{MSC}$ in the control group, and $5 * 10^{6} \mathrm{MNC}$ in the buffy coat group to ensure the same total number of cells used for comparison. The concentration of MSC in bone marrow extracted from rabbit iliac crest was previously reported equal to $607.8 / \mathrm{ml}$ [9]. Thus, there are approximately $1216 \mathrm{MSC}$ and $4.6 \times 10^{6} \mathrm{MNCs}$ in $2 \mathrm{~mL}$ of bone marrow. After PGA plus HA scaffold, the buffy coat was easily fixed on the defected cartilage. We used PGA to cover cartilage defects, similar to a membrane. And it was disclosed that cartilage regeneration is generally stable and the appearance is acceptable after coverage of PGA. Siciari et al. demonstrated that a cell-free PGA-HA scaffold has an appropriate cartilage regeneration effect.[35]

Our in vivo study revealed that some individuals with poor cartilage regeneration had hyper osteogeny around the joints and had traumatic arthritis as well. These may be related to surgery or individual differences. However, the cartilage regeneration effect in buffy coat+HA group was found to be very satisfactory and stable. When the bone marrow was pumped out for a long period of time, the bone marrow was easily solidified in the syringe. When the buffy coat was extracted with such bone marrow, the buffy coat likely contained several RBCs or MSCs. The same situation was also observed when the ambient temperature was high.

However, the buffy coat was extracted by Ficoll gradient centrifugation, Ficoll couldn't be used in clinical practice. Thus, it is essential to develop a new method for extracting buffy coat over the Ficoll gradient centrifugation, which is the key to the application of buffy coat in the future.

It turns out that buffy coat does have a positive effect on cartilage regeneration. In order to explore the mechanism of cartilage regeneration in buffy coat, this paper elaborates it from the perspective of growth factors. Admittedly, in addition to these factors, there may be other factors that we did not find. Other factors, such as the presence of multiple monocytes in the Buffy coat, and the effect of these cells on MSC is unknown. The study of function of these factors will be the direction of our research in the future.

\section{Conclusions}

In summary, a number of components of buffy coat were herein studied, and it was revealed that there are several growth factors and cytokines that promote cartilage regeneration. The capability of buffy coat to produce cartilage was also demonstrated in in vitro and in vivo experiments. The problem that the buffy coat is difficult to fix onto the cartilage defect due to the liquid state was solved, and the cartilage regeneration effect in the buffy coat+HA group was verified as well. The clinical application of buffy coat is another main challenge in the treatment of cartilage defect. 


\section{Abbreviations}

BMC: bone marrow concentration;

BMSC: bone marrow mesenchymal stem cell;

DMB: 1,9-Dimethylmethylene Blue;

ECM: extracellular matrix; PRP: Platelet-rich plasma;

FBS: fetal bovine serum;

EGF: epidermal growth factor;

ELISA: enzyme-linked immunosorbent assay;

FGF-2: fibroblast growth factor 2;

GAG: Glycosaminoglycan;

HA: hyaluronic acid;

ICRS score: International Cartilage Repair Societ score;

IGF-1: insulin-like growth factor;

IL-1ra: interleukin 1 receptor antagonist;

IL-1 $\beta$ : interleukin-1 $\beta$;

IL-4: interleukin-6;

IL-6: interleukin-6;

IL-10: interleukin-10;

LSD: least-significant difference;

MNC: mononuclear cell;

MSC: mesenchymal stem cell;

OA: osteoarthritis;

one-way ANOVA: one-way analysis of variance;

PDGF: platelet derived growth factor; 
PGA: polyglycolic acid;

RBC: red blood cell;

TGF- $\beta 1$ : Transforming Growth Factor $\beta 1$;

TNF-a: tissue necrosis factor $a$;

VEGF: vascular endothelial growth factor;

a-MEM: a-minimum essential medium;

\section{Declarations}

\section{Acknowledgement}

The author thank Prof. Xun Cui, Prof. Delai Qiu from Yanbian University and Prof. Lin Li, Prof. Bairi Cui from Yanbian Hospital for their help.

\section{Authors' contributions}

Hang and Guo conducted experiments, prepared manuscripts, and participated in the design, coordination, review and editing of the study. Kumar contributed to the conception and supervision of the paper and made important suggestions for improving the manuscript. Hang and Guo contributed equally to the study. Hao and Shouyong helped to draft the manuscript and drew a graphic abstract. Guangwen and Xu contributed to data interpretation and manuscript preparation. Longhao Jin was the study's instructor and helped design the experiment. All authors read and approve the final manuscript.

\section{Corresponding authors}

Correspondence to Longhao Jin.

\section{Funding}

This study was supported by the grant of the National Natural Science Foundation of China (81560353); China Postdoctoral Science Foundation (2016M601394); Jilin Postdoctoral Science Foundation, China; The Science and Technology Research Project of the Department of Education, Jilin, China.

\section{Availability of data and materials}

The authors confirm that the data supporting the findings of this study are available within the article. 


\section{Ethics approval and consent to participate}

The experimental procedure was approved by the Institutional Animal Care and Use Committee of Yanbian University (Approval No. 2015036).

\section{Consent for publication}

Not applicable.

\section{Competing interests}

The authors declare no competing interest.

\section{Author details}

1. Department of Orthopedic Surgery, Yanbian University Hospital, Yanji City, China.

2. Department of Orthopedic Surgery, Community Hospital, Itahari, Nepal.

${ }^{*}$ Co-first author. These authors contributed equally to this work.

\#Address correspondence and reprint requests to: LongHao Jin; M.D., Ph.D..

Department of Orthopedic Surgery, Yanbian University Hospital.

\section{References}

1. Zhang $\mathrm{L}, \mathrm{Hu} \mathrm{J}$, Athanasiou KA. The role of tissue engineering in articular cartilage repair and regeneration. Crit Rev Biomed Eng. 2009;37:1-57.

2. Andy G, Katrina M, Julian S, Kim L, Razi Z. The use of mesenchymal stem cells for cartilage repair and regeneration: a systematic review. J Orthop Surg Res. 2017 Mar 9;12(1):39.

3. Chang F, Ishii T, Mishima H, Akaogi H, Ogawa T, Ochiai N. Repair of large full-thickness articular cartilage defects by transplantation of autologous uncultured bone-marrow-derived mononuclear cells. J Orthop Res. 2008;26:18-26.

4. Karatoprak O, Korkmaz MF, Kara AN, Gogus A, Isiklar ZU. Early results of autologous mononuclear bone marrow cell implantation in nontraumatic avascular necrosis of the femoral head. Acta Orthop Traumatol Turc. 2008;42:178-183.

5. Ohnishi S, Yasuda T, Kitamura S, Nagaya N. Effect of hypoxia on gene expression of bone marrowderived mesenchymal stem cells and mononuclear cells. Stem Cells. 2007;25:1166-1177. 
6. Yip HK, Chang LT, Wu CJ, Sheu JJ, Youssef AA, et al. Autologous bone marrow-derived mononuclear cell therapy prevents the damage of viable myocardium and improves rat heart function following acute anterior myocardial infarction. Circ J. 2008;72:1336-1345.

7. Bobacz K, Erlacher L, Smolen J, Soleiman A, Graninger WB. Chondrocyte number and proteoglycan synthesis in the aging and osteoarthritic human articular cartilage. Ann Rheum Dis. 2004;63:16181622.

8. Miyazawa K, Shinozaki M, Hara T, Furuya T, Miyazono K. Two major Smad pathways in TGF-beta superfamily signalling. Genes Cells. 2002;7(12):1191-1204.

9. Mariani E, Pulsatelli L, Facchini A. Signaling pathways in cartilage repair. Int J Mol Sci. 2014;15(5):8667-8698.

10. Jin LH, Choi BH, Kim YJ, Park SR, Jin CZ, Min BH. Implantation of bone marrow-derived buffy coat can supplement bone marrow stimulation for articular cartilage repair. Osteoarthritis Cartilage. 2011;19:1440-1448.

11. Jin LH, Zhang ZY, Liu YQ, Kumar Gurung. Comparison of Chondrogenic Ability between Mesenchymal Stem Cells and buffy coat in vitro. Journal of Biosciences and Medicines. 2018;6:8694.

12. Wisniewski HG, Hua JC, Poppers DM, Naime D, Vilcek J, Cronstein BN. TNF/IL-1-inducible protein TSG-6 potentiates plasmin inhibition by inter-alpha-inhibitor and exerts a strong anti-inflammatory effect in vivo. J Immunol. 1996;156:1609-1615.

13. Holmes MW, Bayliss MT, Muir H. Hyaluronic acid in human articular cartilage. Age-related changes in content and size. Biochem J. 1988;250:435-441.

14. Dicker KT, Gurski LA, Pradhan-Bhatt S, Witt RL, Farach-Carson MC, Jia X. Hyaluronan: a simple polysaccharide with diverse biological functions. Acta biomater. 2014;10:1558-1570.

15. Hong HJ, Lee JS, Choi JW, Min BH, Lee HB, Kim CH. Transplantation of autologous chondrocytes seeded on a fibrin/hyaluronan composite gel into tracheal cartilage defects in rabbits: preliminary results. Artif organs. 2012;36 (11): 998-1006.

16. Yang SS, Jin LH, Park SH, Kim MS, Kim YJ, et al. Extracellular Matrix (ECM) Multilayer Membrane as a Sustained Releasing Growth Factor Delivery System for rhTGF- $\beta 3$ in Articular Cartilage Repair. PLoS One. 2016; 11(6): e0156292.

17. William CG, Kim TK, Taboas A, Malik A, Manson P, et al. In vitro chondrogenesis of bone marrowderived mesenchymal stem cells in a photopolymerizing hydrogel. Tissue Eng. 2003 Aug;9(4):67988.

18. Kilkenny C, Browne WJ, Cuthill IC, Emerson M. Altman, DG. Improving bioscience research reporting: the ARRIVE guidelines for reporting animal research. Osteoarthritis Cartilage. 2012;20:256-260.

19. Grogan SP, Barbero A, Winkelmann V, Rieser F, Fitzsimmons JS, O'Driscoll S, et al. Visual histological grading system for the evaluation of in vitro generated neocartilage. Tissue Eng. 2006;12:2141-2149.

20. Cui JH, Park SR, Park K, Choi BH, Min BH. Preconditioning of mesenchymal stem cells with lowintensity ultrasound for cartilage formation in vivo. Tissue Eng. 2007;13:351-360. 
21. Roman-Blas JA, Stokes DG, Jimenez SA. Modulation of TGF-beta signaling by pro-inflammatory cytokines in articular chondrocytes. Osteoarthritis Cartilage. 2007;15:1367-1377.

22. Castillo TN, Pouliot MA, Kim HJ, Dragoo JL. Comparison of growth factor and platelet concentration from commercial platelet-rich plasma separation systems. Am J Sports Med. 2011;39(2):266-271.

23. Veselý D, Astl J, Lastůvka P, Matucha P, Sterzl I, Betka J. Serum levels of IGF-I, HGF, TGFbeta1, bFGF and VEGF in thyroid gland tumors. Physiol Res. 2004;53(1):83-89.

24. Maehara H, Sotome S, Yoshii T, Torigoe I, Kawasaki Y, Sugata Y, et al. Repair of large osteochondral defects in rabbits using porous hydroxyapatite/collagen ( $\mathrm{HAp} / \mathrm{Col}$ ) and fibroblast growth factor-2 (FGF-2). J Orthop Res. 2010;28:677-686.

25. Lara L, Lynda O, Srikanth A, Brian J, Kimberly S, et al. Effect of IGF-I in the Chondrogenesis of Bone Marrow Mesenchymal Stem Cells in the Presence or Absence of TGF- $\beta$ Signaling. J Bone Miner Res. 2006 Apr;21(4):626-36.

26. Schmidt MB, Chen EH, Lynch SE. A review of the effects of insulin-like growth factor and platelet derived growth factor on in vivo cartilage healing and repair. Osteoarthritis and Cartilage. 2006;14:403-412.

27. Dhillon RS, Schwarz EM, Maloney MD, Platelet-rich plasma therapy - future or trend? Arthritis Res Ther. 2012;14(4):219.

28. Shepard JB, Jeong JW, Maihle NJ, O'Brien S, Dealy CN. Transient anabolic effects accompany epidermal growth factor receptor signal activation in articular cartilage in vivo. Arthritis Res Ther. 2013;15(3):R60.

29. Schuerwegh AJ, Dombrecht EJ, Steven WJ, van Offel JF, Bridts CH, De Clerck LS. Influence of proinflammatory (IL-1, IL-6, TNF-a, IFN- $\gamma$ ) and anti-inflammatory (IL-4) cytokines on chondrocyte function. OsteoArthritis and Cartilage. 2003;11(9):681-687.

30. Behrendt P, Feldheim M, Preusse-Prange A, Weitkamp JT, Haake M, Eglin D, et al. Chondrogenic potential of IL-10 in mechanically injured cartilage and cellularized collagen ACl grafts. Osteoarthritis and Cartilage. 2018;26:264-275.

31. van Meegeren ME, Roosendaal G, Jansen NW, Wenting MJ, van Wesel AC, van Roon JA, et al. IL-4 alone and in combination with IL-10 protects against blood-induced cartilage damage. Osteoarthritis and Cartilage. 2012;20(7):764-772.

32. Hou JZ. Bone marrow PRP intra-articular injection of knee osteoarthritis observation. Journal of Transportation Medicine. 2016;2(1):73-75.

33. Horn P, Bork S, Wagner W. Standardized Isolation of Human MesenchymalStromal Cells with Red Blood Cell Lysis. Methods in Molecular Biology. 2011;698:23-35.

34. Cassano JM, Kennedy JG, Ross KA, Fraser EJ, Goodale MB, Fortier LA. Bone Marrow Concentrate and Platelet-Rich Plasma Differ in Cell Distributionand Interleukin 1 Receptor Antagonist Protein Concentration. Knee Surgery Sports Traumatology Arthroscopy. 2016;26:333-342.

35. Siclari A, Mascaro G, Gentili C, Cancedda R, Bouxr E. A cell-free scaffold-based cartilage repair provides improved function hyaline-like repair at one year. Clin Orthop Relat Res. 2012;470(3):910- 
Figures

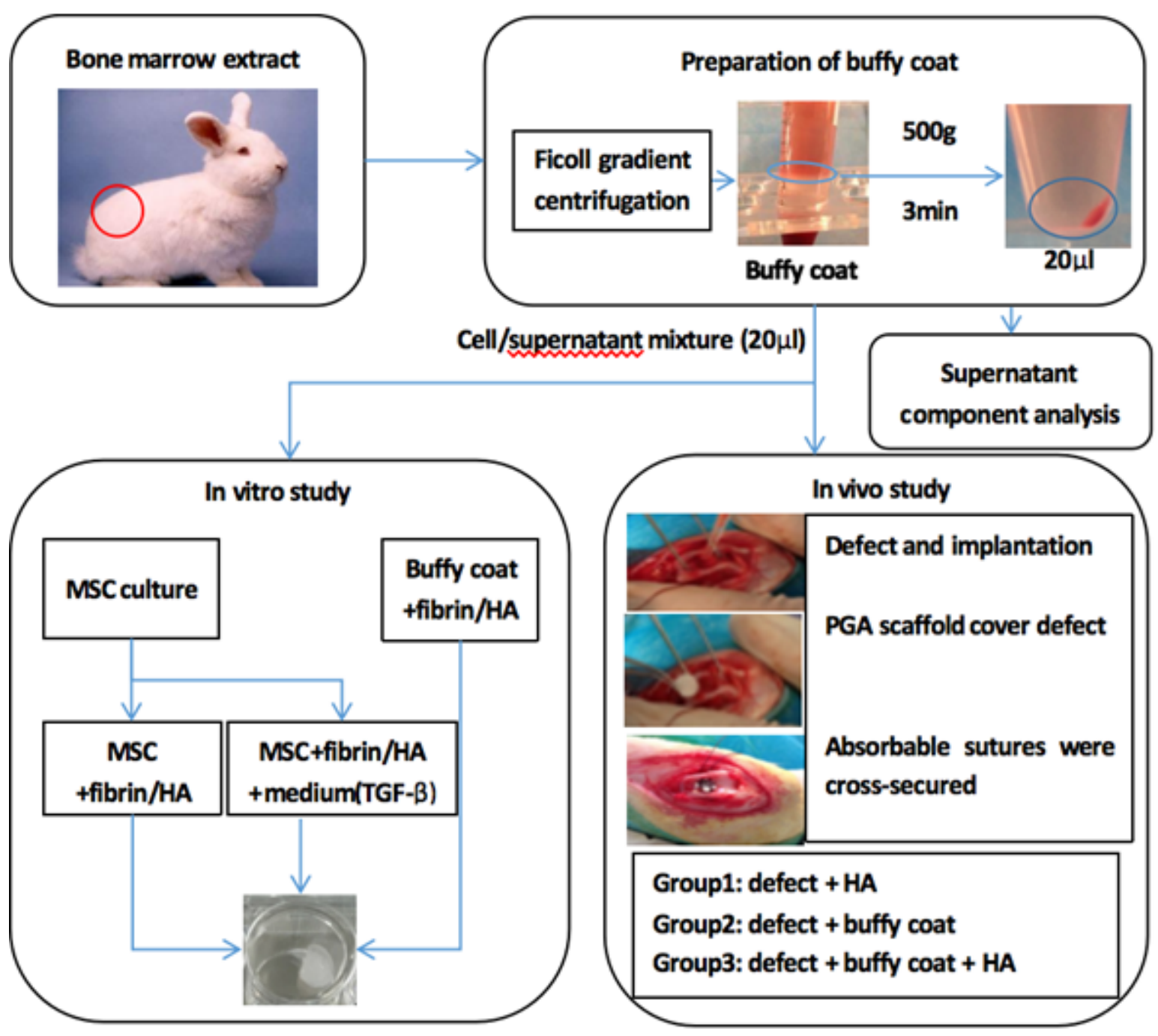

Figure 1

$2 \mathrm{ml}$ bone marrow was extracted from the anterior superior iliac crest of rabbits using a $20 \mathrm{ml}$ syringe containing $2 \mathrm{ml}$ of $0.9 \%$ sodium chloride solution. The bone marrow was placed in a tube containing Ficoll, and centrifuged at $1000 \mathrm{~g}$ for $30 \mathrm{~min}$ at $4{ }^{\circ} \mathrm{C}$. We extract $0.5 \mathrm{ml}$ buffy coat into $1.5 \mathrm{ml}$ tube and centrifuge again at $500 \mathrm{~g}$ at $4{ }^{\circ} \mathrm{C}$ for $3 \mathrm{~min}$. $20 \mathrm{ul}$ cells and supernatant mixture were extracted for in vitro and in vivo experiments. In vitro experiment, they were divided into 3 groups: MSC group, MSC+TGF- $\beta$ group, and buffy coat group. In vivo experiments, they were divided into 3 groups: MSC+HA group, buffy coat group, and buffy coat+HA group. 


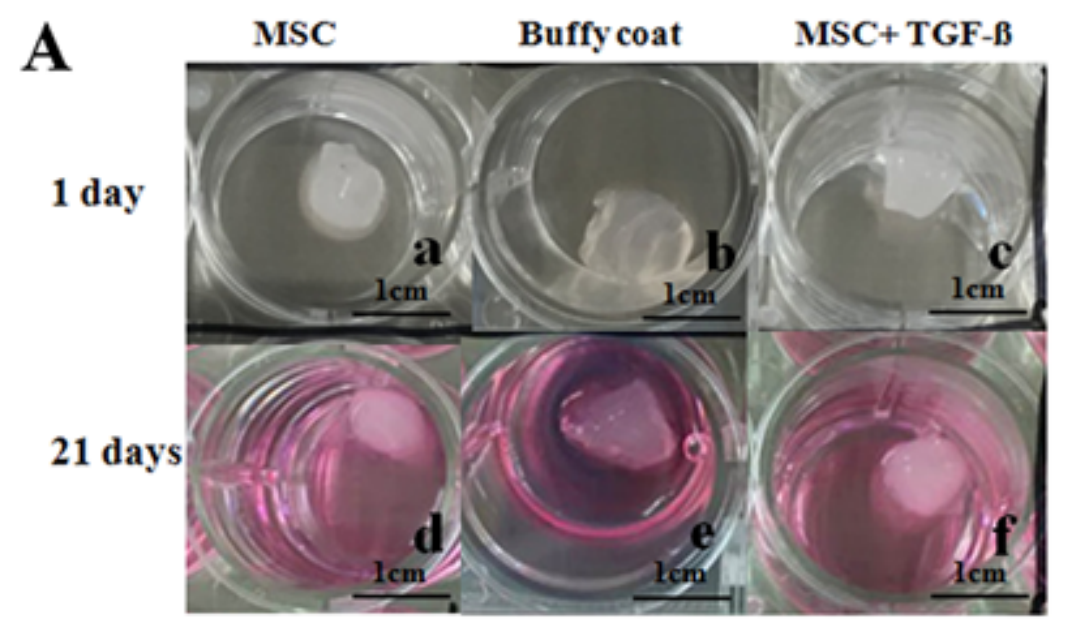

B

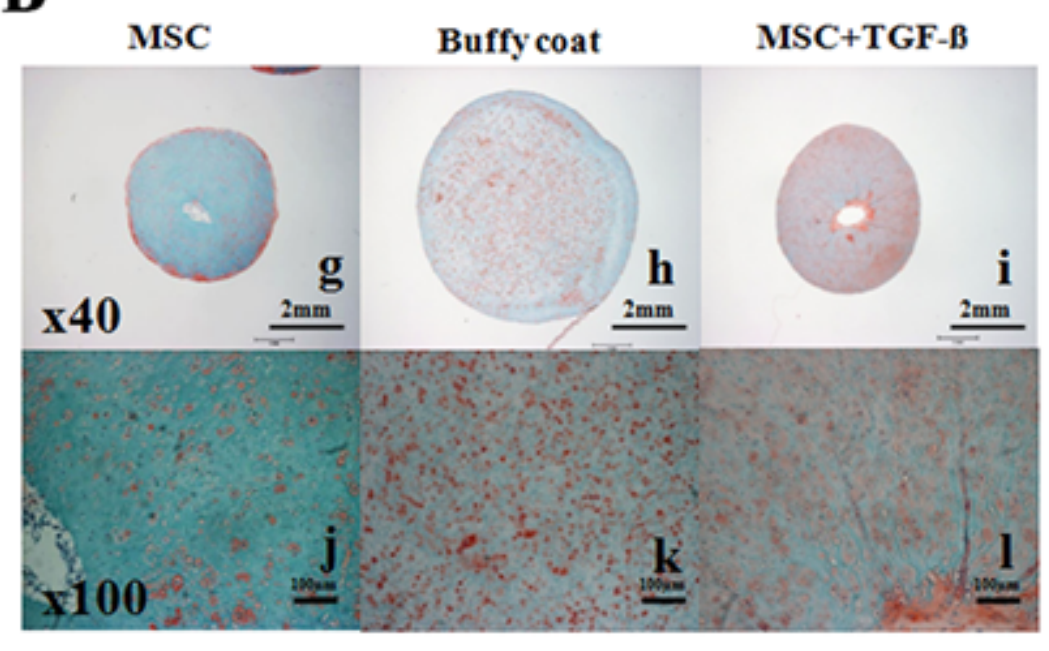

Figure 2

(A) shows the gross image after 1 and 21 days. a-c illustrate the image after cell inoculation, and there is basically no difference in size after the three groups are inoculated; d-f display the image after 21 days of culture. $d$ shows that the size is small and the edges are blurred; the size of e and $f$ is basically unchanged, and the edges are round and clear. The red liquid is chondrogenic defined medium. (B) depicts staining with Safranin-O after 21 days; $\mathrm{g}-\mathrm{i}$ display magnification at $\mathrm{X} 40$, and $\mathrm{j}-1$ illustrate magnification at X100. There is a limited red area around $\mathrm{g}$, and a hole in the center. In $\mathrm{h}$ and $\mathrm{I}$, the red dotted area is evenly distributed, and a hole appears in the central area of $\mathrm{i}$. 


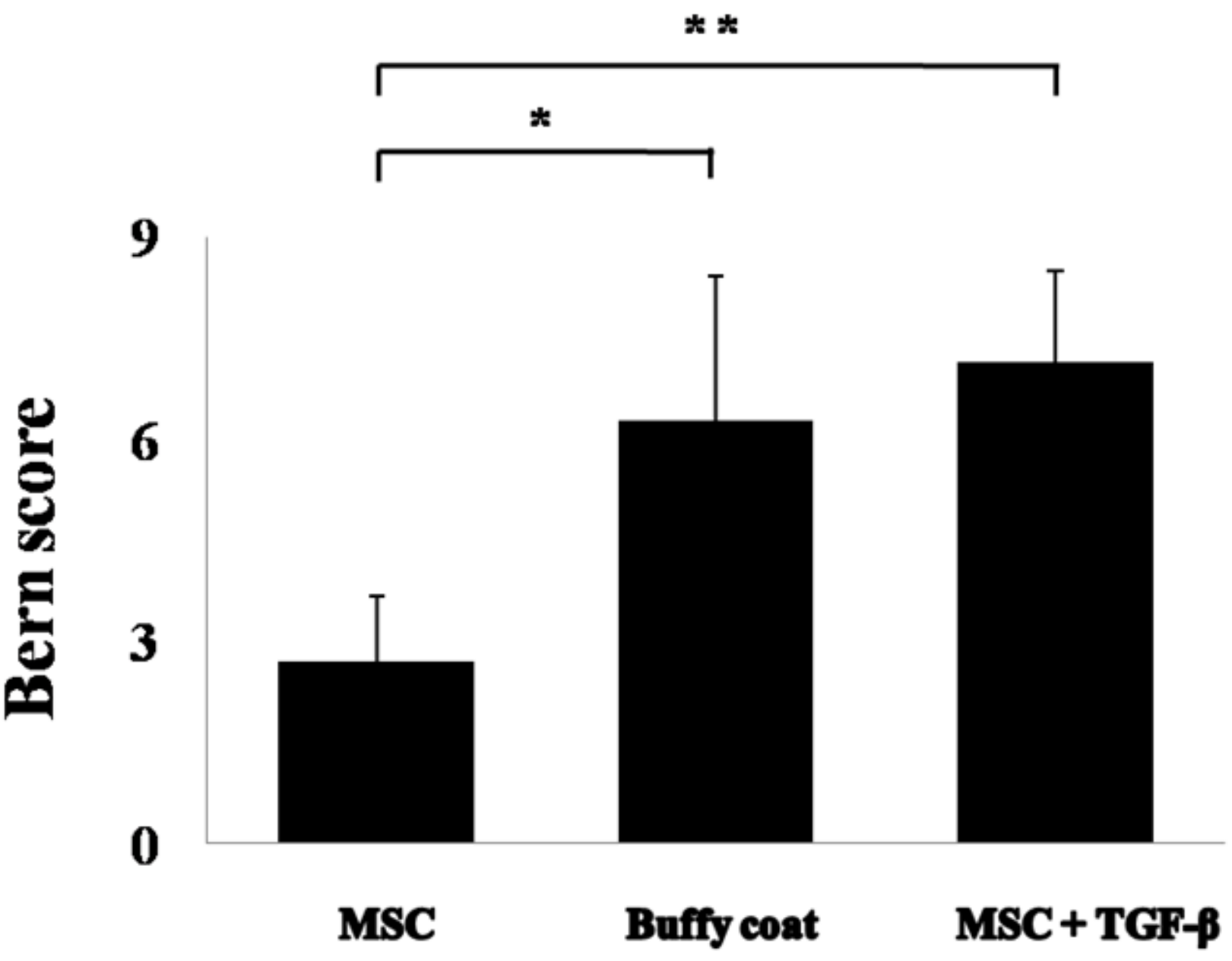

Figure 3

Bern score of in vitro study after 21 days. There was a statistical gap between MSC and Buffy coat group. There was a statistical difference between MSC and MSC+TGF- $\beta$ group $(n \geq 4 ; * p<0.05 ; * \star p<0.01)$. 

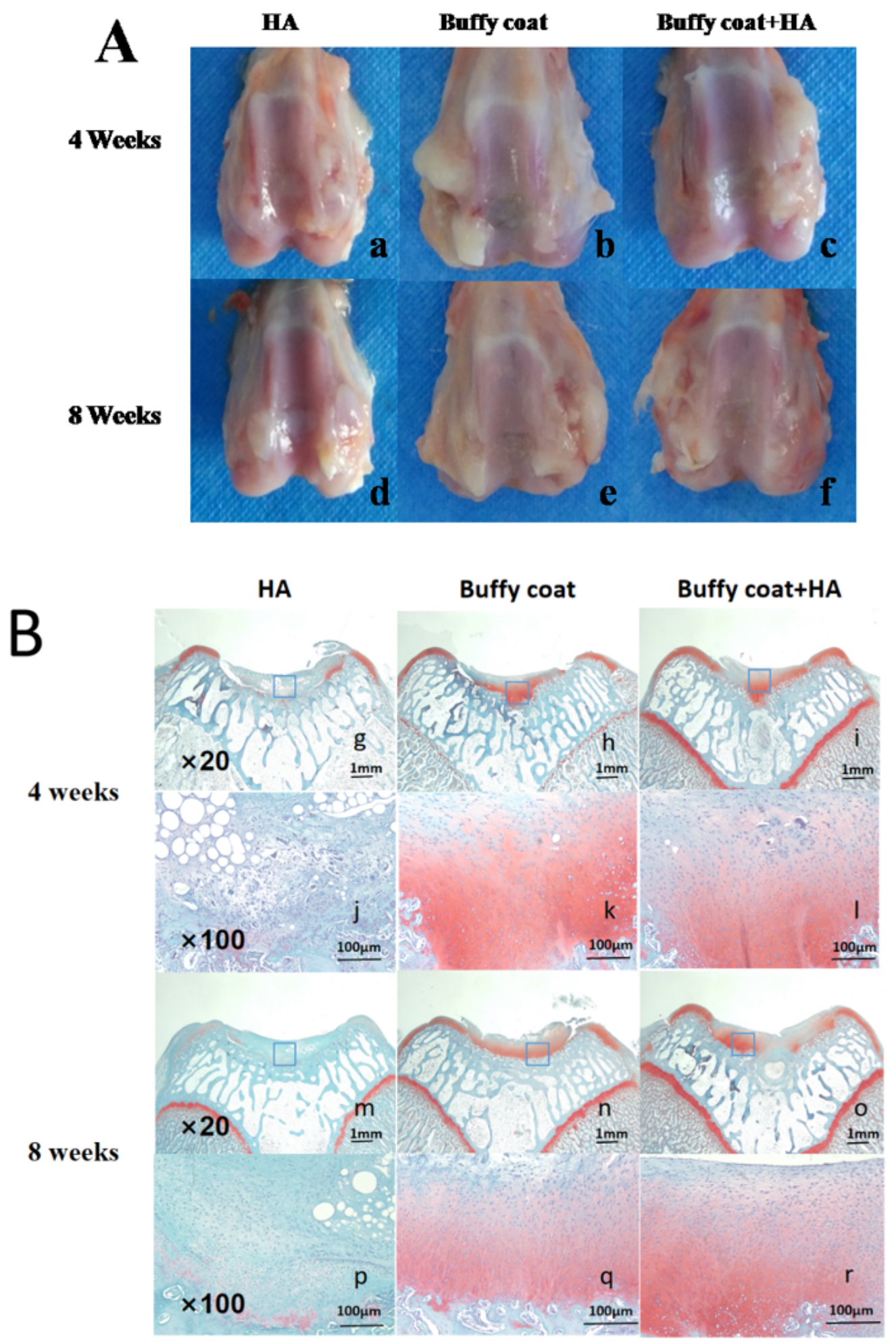

\section{Figure 4}

(A) a-c are images of the knee joint at the 4th week after surgery; various levels of regenerative tissue can be observed. a small amount of tissue is formed, $b$ and $c$ regenerative tissue covers the cartilage defect; d-f are images of the knee joint at the 8th week after surgery, showing that the cartilage defect area is completely covered by regenerated tissue. (B) g-l shows staining with Safranin-0 at the 4th week after operation, no dark red area was observed in the HA group, and the regenerated tissue in the Buffy coat 
and Buffy coat+HA groups was evenly stained dark red; 0 staining, there were very few dark red areas in the HA group, and the Buffy coat and Buffy coat+HA groups showed mature regenerated tissue.

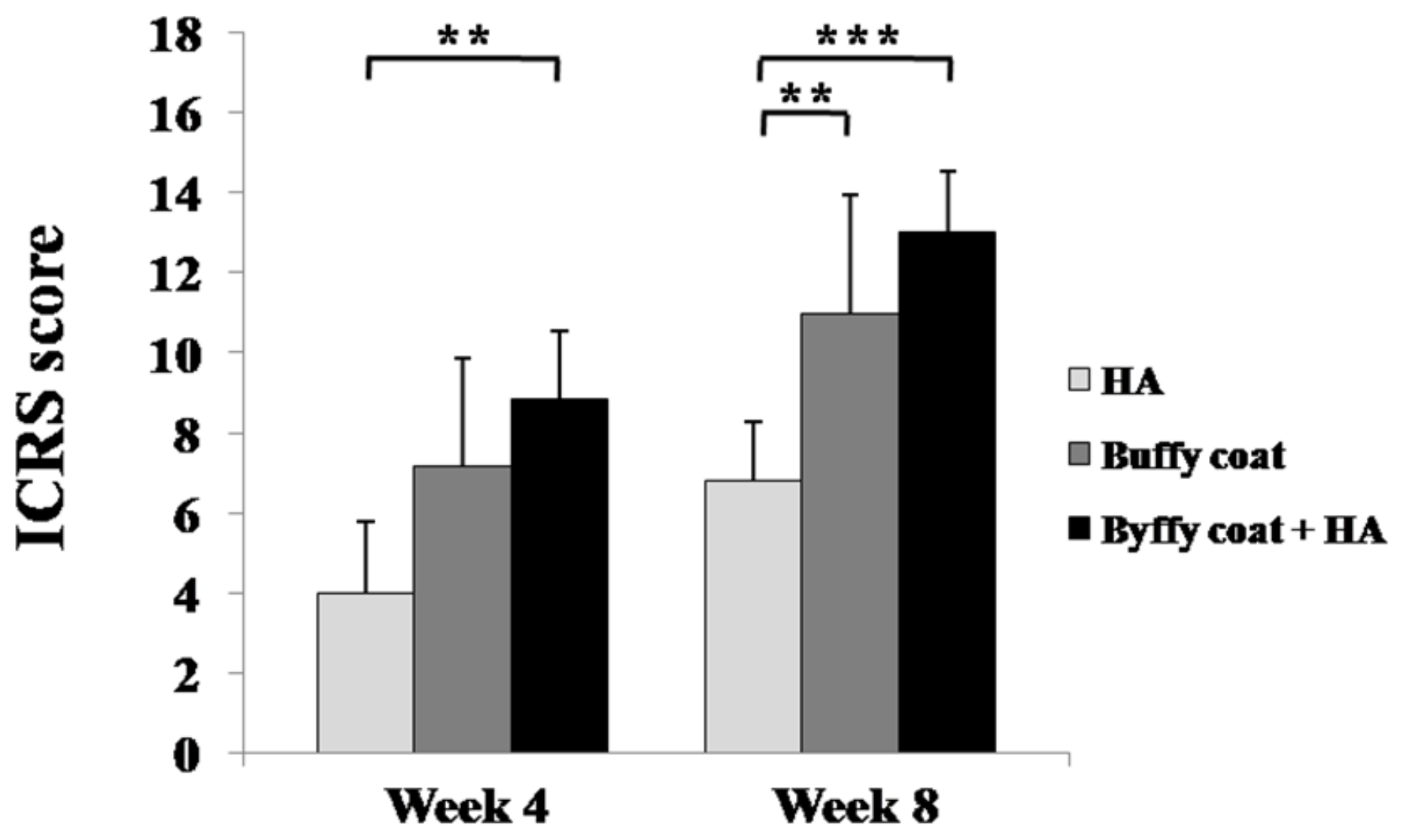

Figure 5

ICRS scores at 4th and 8th weeks post-operation. The ICRS scores significantly increased in all the groups over time. There was a statistically significant difference in the scores between the HA group and the buffy coat+HA group at 4th week. At 8th week, there was a gap between the scores of HA group and buffy coat group, and the scores in HA and buffy coat+HA groups were larger $(n \geq 5 ; * \star p<0.01$; $\star \star \star p<0.001)$. 


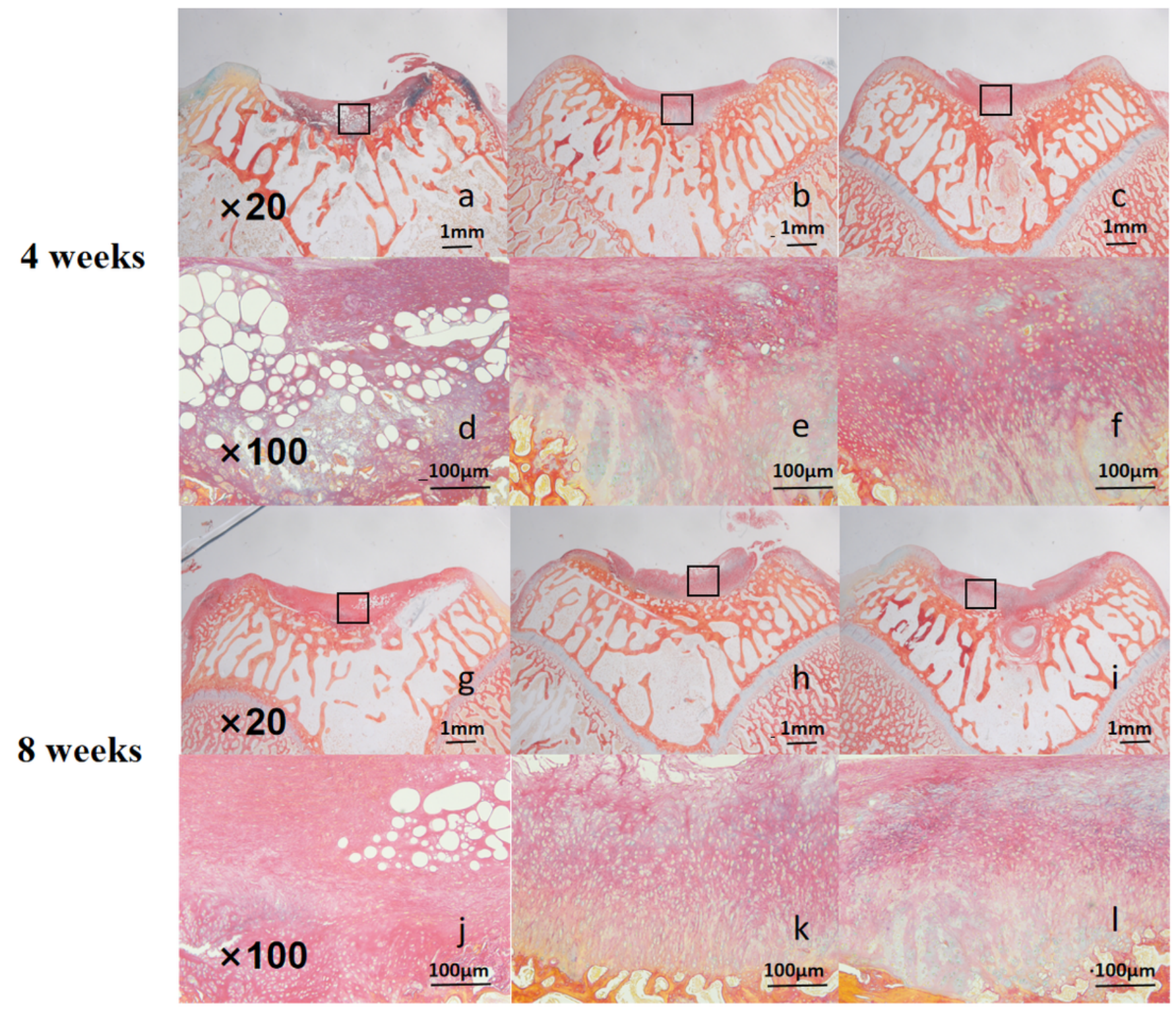

\section{Figure 6}

a-f shows Sirius Red staining at the 4th week after operation. The collagen fibers regenerated in the HA group (a, d) were very small, while the Buffy coat group (b, e) and Buffy coat+HA (c, f) have relatively more collagen fibers, but the regenerated collagen fibers in each group were irregularly arranged. $\mathrm{g}-\mathrm{l}$ is Sirius Red staining at the 8th week after operation, showing that the regenerated collagen fibers in the HA group $(g, j)$ were very thin, while mature collagen fibers appeared in Buffy coat group $(h, k)$ and Buffy coat+HA group $(\mathrm{i}, \mathrm{l})$. 


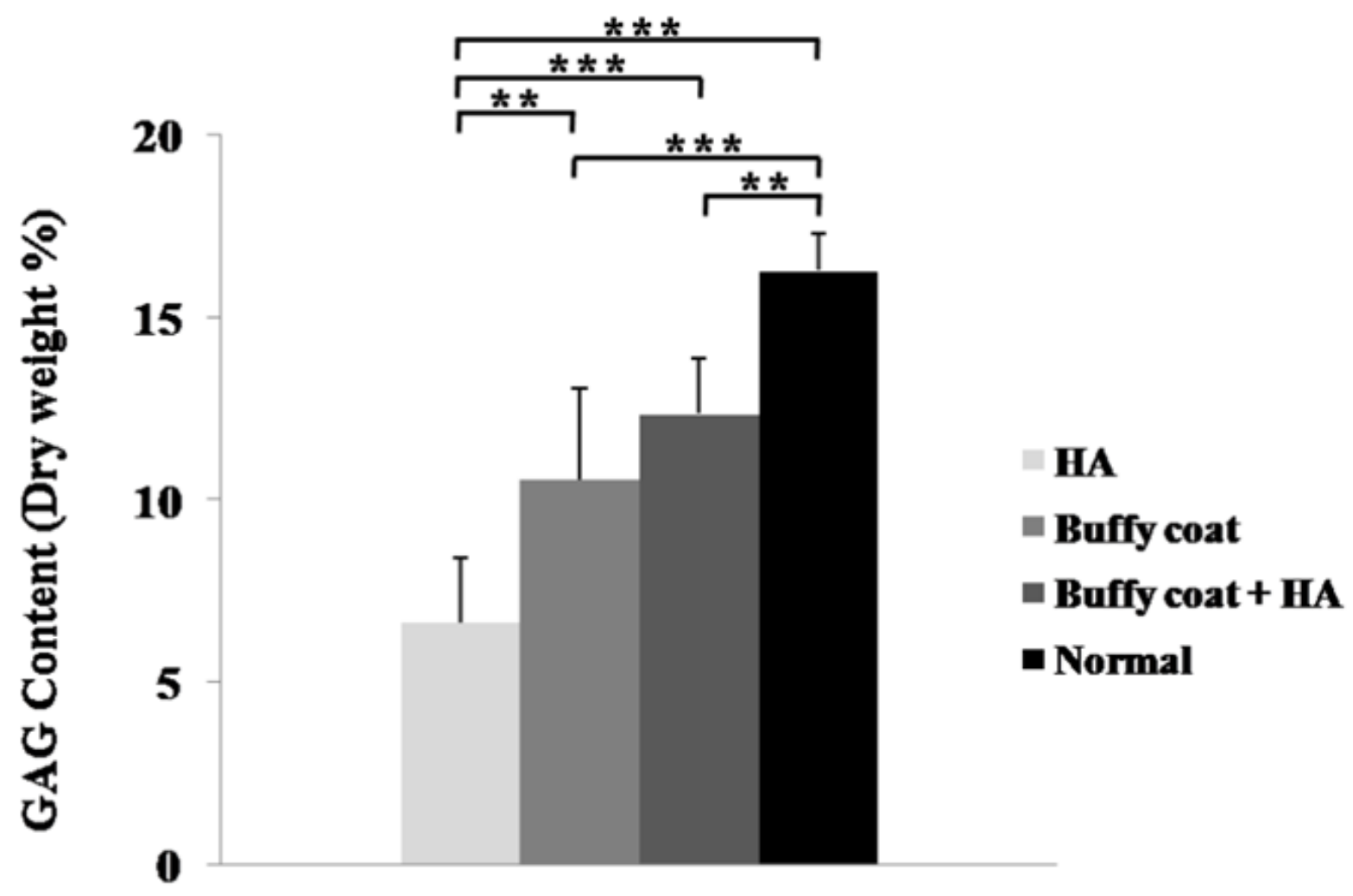

Figure 7

It is the percentage of GAG content (dry weight) $\left(n=6 ;{ }^{* \star} p<0.01 ; * \star \star p<0.001\right)$. 